\title{
REALIZATION OF AN INERTIAL REFERENCE FRAME FROM MARK III VLBI
}

\author{
C. MA \\ Goddard Space Flight Center \\ Geodynamics Branch \\ Greenbelt, Maryland 20771 \\ USA
}

\begin{abstract}
Over 350000 dual frequency Mark III VLBI observations from several geodetic and astrometric observing programs have been used to realize an inertial reference frame through the positions of 325 compact extragalactic radio sources uniformly distributed over the sky with standard errors typically under 1 milliarcsecond (mas). Internal and external tests indicate that the reference frame defined by the relative positions of these radio sources should be accurate and stable at the 1-2 mas level. Because the conventional precession and nutation models are adjusted in the estimation of the source positions, the positions and relative angles are not degraded over the interval of observations or at epochs away from the reference epoch.
\end{abstract}

\section{Introduction}

Compact extragalactic radio sources can be used to define a kinematically fixed reference frame against which precise measurements of celestial and terrestrial motions can be made. This paper describes the results obtained from the analysis of ten years of Mark III VLBI data acquired by several geodetic and astrometric observing programs. The important features of the Mark III VLBI system applicable to high precision astrometry are reviewed. The implications of the currently used method of analysis are discussed. In addition to describing the current status of catalog distribution and precision, several sources of systematic error are considered. The future prospects of the radio reference frame are forecast.

There are several papers developing precise radio catalogs from VLBI observations (Fanselow $e t$ al., 1984; Ma et al., 1986; Robertson et al., 1986; Sovers et al., 1988; Ma et al., in press; Sovers, this volume). This paper extends the previous catalogs through new observing programs designed to fill gaps in the surveyed sky and through further extension of the geodetic VLBI data base. This paper is, however, preliminary in nature, and the details of the new data will be described elsewhere (Russell et al., in preparation; Ma et al., in preparation).

\section{Technique}

VLBI observations for high precision geodesy and astrometry rely on certain features of the Mark III VLBI system which are not commonly used in astronomical VLBI. These are given in Table 1.

There are two simultaneous observing frequencies to allow calibration of the ionosphere. The 400 $\mathrm{MHz}$ receiver bandwidth at the primary observing frequency allows precise determination of the 
Table 1. Mark III VLBI System

\begin{tabular}{llll}
\hline Receiver & Record & Clock & Correlators \\
$8.4 \mathrm{Ghz}-400 \mathrm{MHz}$ & 28 tracks & Maser & Haystack \\
$2.3 \mathrm{GHz}-80 \mathrm{MHz}$ & $2 \mathrm{MHz} /$ track & & $\begin{array}{l}\text { Bonn } \\
\text { Washington } \\
\end{array}$ \\
& 12 passes/tape & & Kashima \\
\hline
\end{tabular}

group delay observable. The recording of 14 simultaneous tracks (reduced from the 28 possible for operational simplicity) permits both a good delay resolution function and high sensitivity. There have been some experiments carried out where both the receiver bandwidth and the recorded bit rate were doubled. Twelve passes per tape permits considerable savings of tape and transportation costs. The source of time and frequency is always a hydrogen maser. There are four correlator facilities which have contributed to the data set described in this paper. Geodetic observations commonly involve networks of four to seven stations with baselines as short as $1 \mathrm{~km}$ but more commonly 2000 to $6000 \mathrm{~km}$. Some baselines involving South Africa and Australia may be as long as $11000 \mathrm{~km}$. Astrometric networks are generally two or three stations with 3000 to $10000 \mathrm{~km}$ baselines. During a normal 24-hour experiment 20 to 40 sources are observed. Counting an observation as the delay and delay rate pair from a single baseline looking at a single source for 100 to 800 seconds, geodetic sessions usually generate 400 to 1000 observations. The number of observations from astrometric sessions is generally smaller.

\section{Observing Programs and Data}

The geodetic and astrometric observing programs whose data are included in this paper are summarized in Table 2. It should be mentioned that there is another astrometric program run by the Jet Propulsion Laboratory (JPL) using the Deep Space Network stations which parallels certain aspects of the programs used here (Sovers, this volume). Table 2 shows the observing programs, sponsoring organization(s), starting dates, and number of observations, sessions, sources, and sites to 1989.5. The objective of the Crustal Dynamics Project (CDP) is to measure global and regional site velocities while IRIS and NavNet make frequent, evenly spaced measurements of polar motion and UT1. Approximately 50 of the geodetic sources have been used regularly and have at least several hundred observations. The data are stored in the Mark III VLBI data base at Goddard Space Flight Center after being made available by the sponsoring organization.

\section{Data Analysis}

These data were analyzed using the CALC/SOLVE software developed at Goddard. The a priori models include a theoretical VLBI delay (Robertson, 1975), IAU-sanctioned precession and nutation, general relativity (Shapiro, 1967), and tides (both solid Earth and ocean loading). The ionosphere and atmosphere are calibrated using real-time information. Adjusted parameters include source positions, site positions, daily Earth orientation parameters, daily nutation offsets and 
Table 2. Observing Programs and Data to 1989.5

\section{Geodetic:}

Crustal Dynamics Project (NASA-1979)

IRIS (NGS-1980)

NavNet (USNO-1987)
Data:

340000 observations

840 sessions

70 sources

30 sites

12000 observations

40 sessions

\section{Astrometric:}

Crustal Dynamics Project (NASA-1980)

Reference Frame (NRL-1987)

South (NRL, CSIRO, HRAO, CRL-1988)

NASA - National Aeronautics and Space Administration

IRIS - International Radio Interferometric Surveying

NGS - National Geodetic Survey

USNO - United States Naval Observatory

NRL - Naval Research Laboratory

CSIRO - Commonwealth Scientific and Industrial Research Organization (Australia)

HRAO - Hartebeesthoek Radio Astronomy Observatory (South Africa)

CRL - Communication Research Laboratory (Japan)

nuisance parameters such as the clocks and residual atmospheres. See Ma et al. (in press) for further details. The post-fit weighted $\mathrm{rms}$ residual from a solution using all the data is 42 ps while the astrometric data alone fit at $56 \mathrm{ps.}$

It should be emphasized that the adjustment of nutation offsets in longitude and obliquity for each day of data is a departure from conventional practice that is necessary because the intrinsic accuracy of the VLBI data is better than that of the standard precession and nutation models. Herring et al. (1986) demonstrated that the IAU 1980 nutation series has errors at the 1-2 mas level while Sovers et al. (1988) suggested that the precession constant might be in error by as much as $1.6 \mathrm{mas} / \mathrm{yr}$. If the standard precession and nutation models were used without adjustment, the positions of sources observed at only a few, scattered epochs in one year would be affected by the short term nutation errors while the positions of sources observed continually over a long interval or greatly separated in time would be blurred by the errors in the long period nutation terms and the precession constant.

The current practice in VLBI analysis is to adopt the standard IAU precession and nutation models for a reference day, preferably one on which the network is strong and the data of high quality. These models, along with an arbitrary choice of right ascension zero point, define the orientation of the catalog coordinate system with respect to the extragalactic objects. For each other day, a pair of offsets in longitude and obliquity is estimated. The data from different days are combined through the overlap of sources observed, and the actual celestial pole of the catalog is that of the reference day. The mean epoch of the observations therefore loses its conventional meaning. Implicit in this method of analysis are the assumptions that extragalactic objects have negligible proper motions because of their great distance and that source structure is insignificant. Figure 1 shows the adjustments in longitude (scaled by $\sin \varepsilon$ ) and obliquity from 1979-88. The overall slope in longitude 


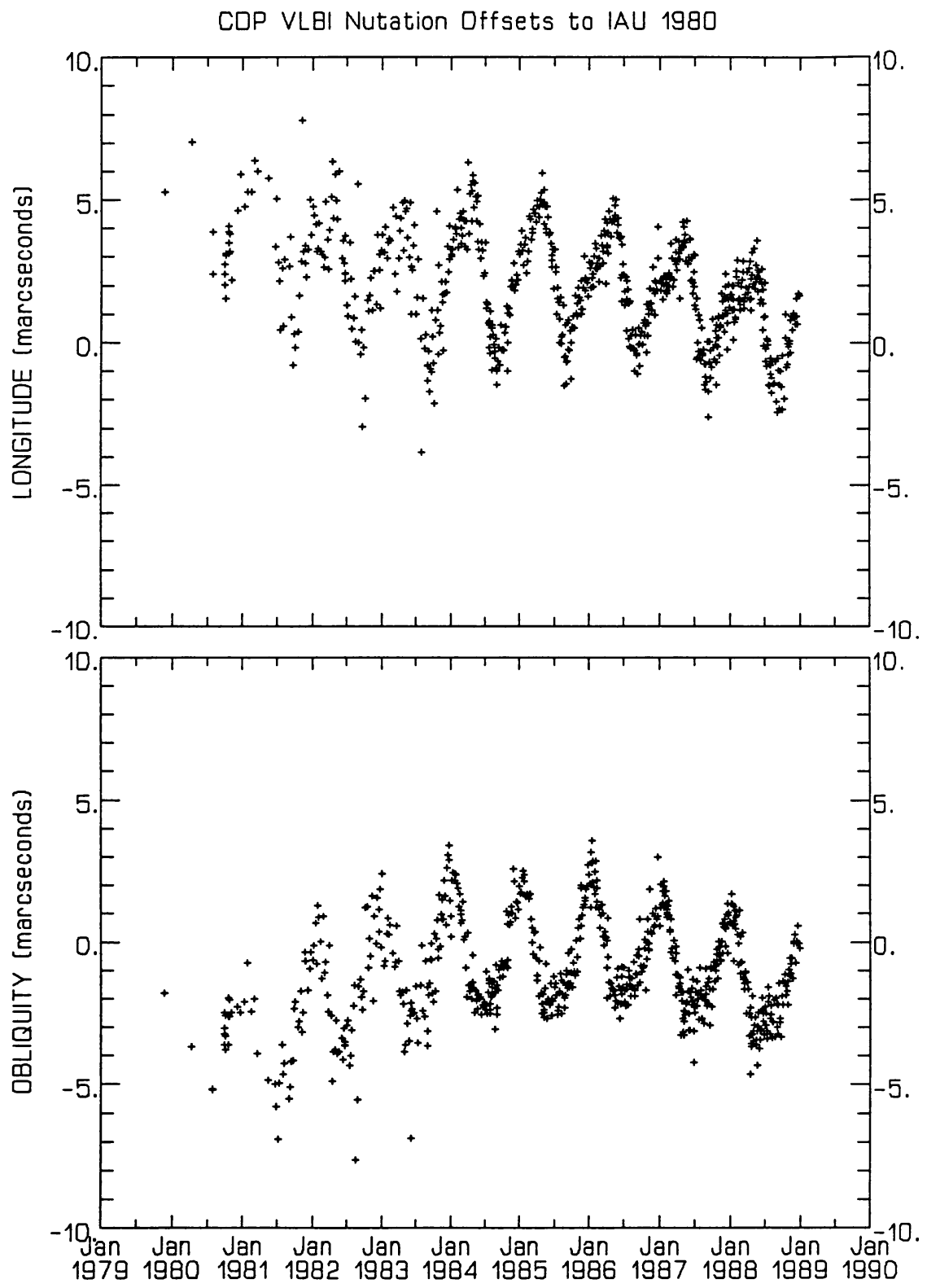

Figure 1. Daily nutation offsets estimated from CDP/RIS VLBI (1979-88). 
is indicative of the error in precession while the curvature in obliquity reflects the error in the principal nutation term. It can be seen that the amplitude of the errors is much larger than the internal error of the VLBI positions (section 5). The adjustment of the precession/nutation model also prevents the degradation of the relative positions at epochs distant from the observations.

\section{Astrometric Results}

The astrometric results include 325 sources with at least one good observation. There are 305 sources with formal standard errors in right ascension and declination less than 5 mas and 224 sources with formal standard errors less than 1 mas. The distribution of the sources is shown in Figure 2 on an equal area projection. Because of the nature and scope of the geodetic programs, most of the 50 best observed sources are in the north although there are some as far south as $-30^{\circ}$. The sources below $-45^{\circ}$ were observed with the Australia-South Africa baseline while the sources between $-45^{\circ}$ and $-30^{\circ}$ were observed with the Australia-Japan-Hawaii network. The distribution of sources by right ascension sector and declination band is shown in Figures 3 and 4 . Figures 5 and 6 show the histograms of formal errors $\sigma(\alpha) \cos \delta$ and $\sigma(\delta)$, respectively, in 0.5 mas bins. The peaks at the right are the remaining outliers. It can be seen that the overall distribution of sources overall is quite uniform and that the distribution of errors is concentrated below 1 mas.

\section{Errors}

While the formal errors are quite small it is instructive to examine the data in different ways to understand the stability of the adjusted positions and the possible systematic errors. Table 3 shows a comparison of the positions from a catalog generated from many years of data with the positions generated from annual solutions. Except for using the same reference day, the annual solutions were completely independent. The same reference day was also used for the multiyear solution.

Table 3. Comparison of 80-87 Catalog with Annual Solutions

\begin{tabular}{lcllllll}
\hline year & $\begin{array}{l}\text { number } \\
\text { sources }\end{array}$ & $\begin{array}{l}\text { number } \\
\text { observations }\end{array}$ & $\begin{array}{l}\mathrm{X} \\
\text { mas }\end{array}$ & $\begin{array}{l}\text { Y } \\
\text { mas }\end{array}$ & $\begin{array}{l}\text { Z } \\
\text { mas }\end{array}$ & $\begin{array}{l}\text { sin } \delta \\
\text { mas }\end{array}$ & $\begin{array}{l}\Delta \delta \\
\text { mas }\end{array}$ \\
80 & 50 & 13000 & -0.1 & 0.2 & -1.3 & -4.0 & 3.8 \\
81 & 49 & 9000 & 0.5 & 0.7 & -1.5 & -0.5 & 1.0 \\
82 & 39 & 13000 & 0.5 & -0.1 & -0.7 & -0.3 & 0.6 \\
83 & 28 & 15000 & 0.3 & -0.3 & -0.6 & 1.0 & -0.4 \\
84 & 53 & 34000 & 0.4 & -0.0 & -0.2 & 0.1 & 0.1 \\
85 & 34 & 47000 & 0.1 & 0.6 & -0.5 & 0.3 & -0.2 \\
86 & 46 & 52000 & -0.1 & -0.1 & 0.5 & -0.1 & -0.1 \\
87 & 145 & 67000 & 0.3 & -0.3 & -0.1 & 0.2 & -0.3
\end{tabular}

$X, Y$, and $Z$ are rotations about orthogonal axes oriented toward $(\alpha, \delta)=(0,0),(6 h r, 0)$ and $\left(0,90^{\circ}\right)$, respectively, between the annual catalog and the multiyear catalog. The last two columns are a systematic difference in declination as a function of declination and an offset in declination, respectively. It can be seen that in the later years, which contribute most to the overall solution, there 


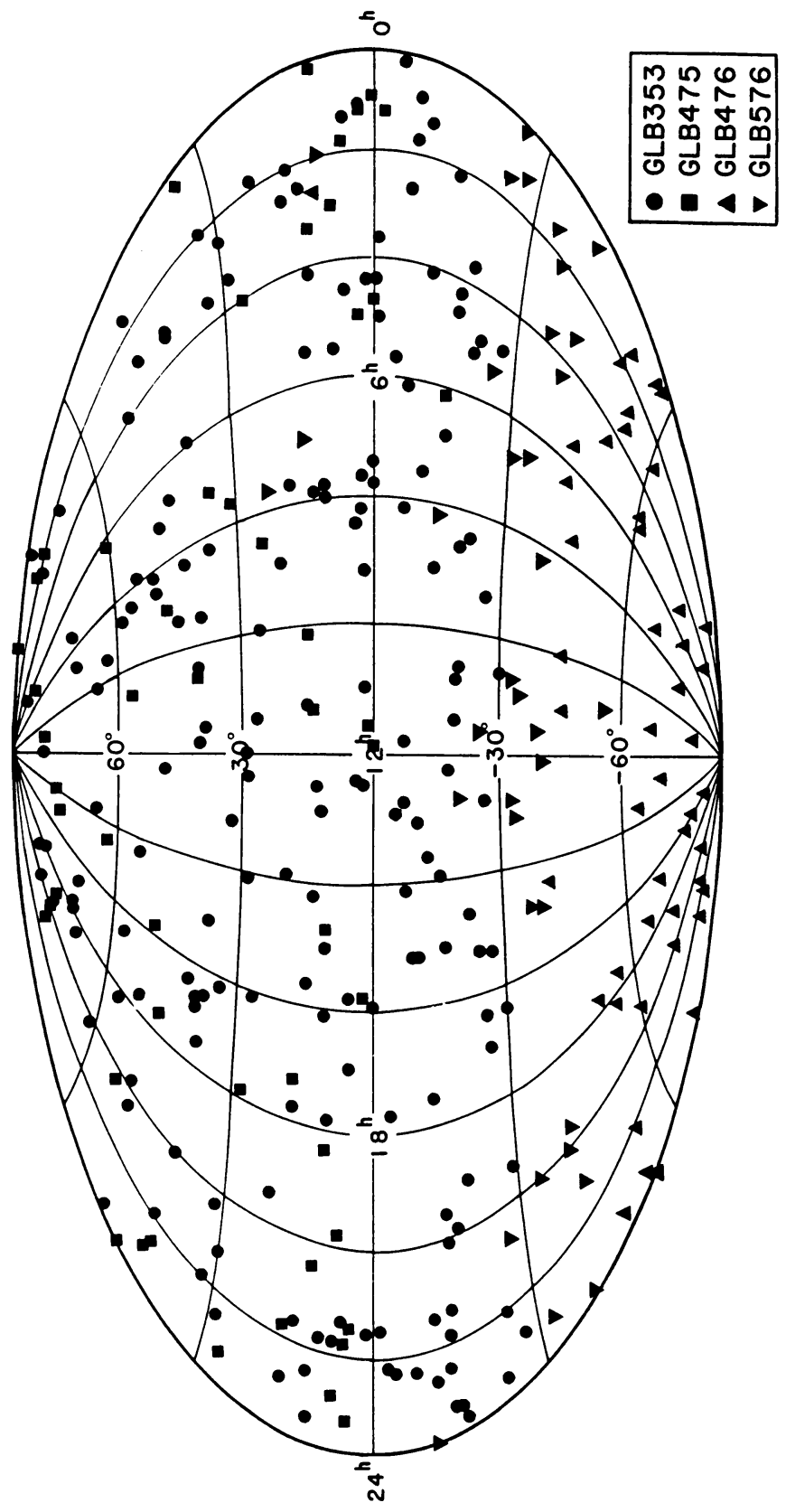

롱

守

宅

ญ्.

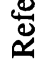

㐘

i

苟

$\ddot{\underline{\Delta}}$

宅

'

ก

ธี

$\dot{8}$

氝

路

옳 돌

กิ

年告

운

苛

造

을

ङ

蛋蛋

is

总 

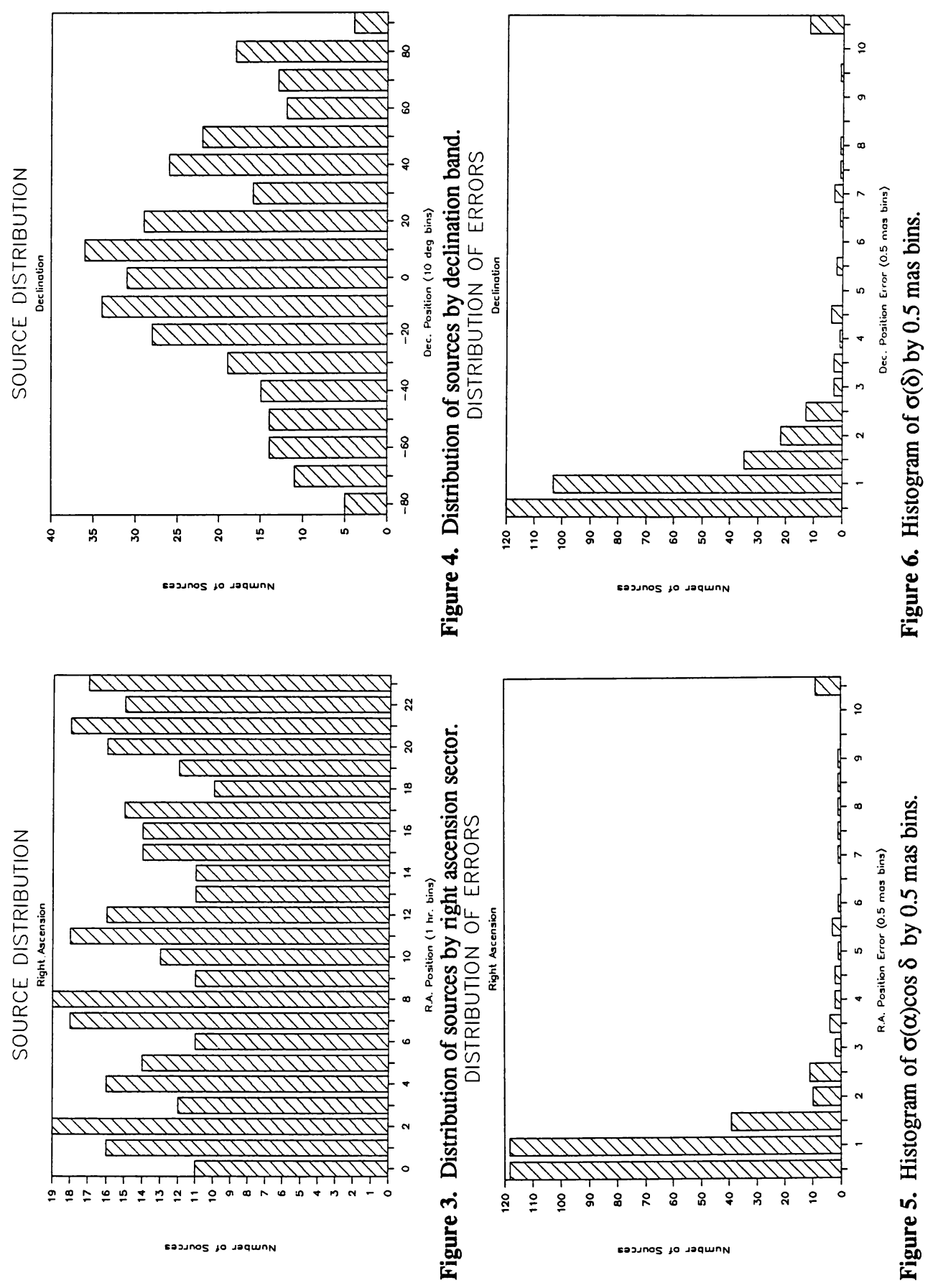
are very small differences between the annual solutions and the full catalog. The larger differences in the earlier years are probably caused by limited network geometry.

Table 4 shows a comparison between the same multiyear catalog and positions from solutions that use data from specific seasons (winter - Dec, Jan, Feb; spring - Mar, Apr, May; summer — Jun, Jul, Aug; fall - Sep, Oct, Nov). Again the same reference day was used for each seasonal solution.

Table 4. Comparison of 80-87 Catalog with Seasonal Solutions

\begin{tabular}{lcrrrrr}
\hline & $\begin{array}{l}\text { number } \\
\text { sources }\end{array}$ & $\begin{array}{c}\mathrm{X} \\
\text { mas }\end{array}$ & $\begin{array}{c}\mathrm{Y} \\
\text { mas }\end{array}$ & $\begin{array}{c}\mathrm{Z} \\
\text { mas }\end{array}$ & $\begin{array}{c}\text { sin } \delta \\
\text { mas }\end{array}$ & $\begin{array}{c}\Delta \delta \\
\text { mas }\end{array}$ \\
Winter & 120 & 0.0 & -0.1 & 0.2 & 0.7 & -0.6 \\
Spring & 106 & -0.3 & 0.5 & -0.0 & -1.1 & 0.9 \\
Summer & 116 & 0.2 & -0.1 & 0.0 & -0.5 & -0.5 \\
Fall & 37 & -0.2 & -0.3 & -0.2 & -1.0 & -0.8 \\
\hline
\end{tabular}

The rotations from the seasonal solutions to the overall catalog are insignificant. There does appear to be some effect on the declinations, which may be caused by seasonal atmospheric differences that are not completely included in the atmospheric calibration and estimation.

A comparison of two completely independent VLBI catalogs is given in Sovers (this volume). The rms position difference of 95 common sources is 2.0 mas in right ascension and 1.3 mas in declination after rotations arising from different reference days and right ascension zero points are removed.

These comparisons illustrate some of the systematic errors which may be present in a radio catalog, although the systematic errors may largely cancel with sufficient data. The distribution of data and networks is not uniform over time or for particular sources. There are correlations between the network geometry and source positions, particularly between baseline $z$-components and declinations, that may affect particular sources. The delay model is another possible source of systematic error, particularly the modeling of general relativistic effects. The International Earth Rotation Service (IERS) standards (McCarthy, 1989) lists several possible algorithms, which may differ in scale or periodic terms. Scale differences will affect baseline lengths and, to some extent, source declinations. The periodic effects are much smaller and are not likely to be visible in the source positions at the present time. Finally, the largest source of noise and possible systematic biases in high precision VLBI is probably the atmosphere. While there have been improvements in both the modeling and estimation of the atmosphere as well as the scheduling of observations to permit better separation of the atmosphere from other effects, the exact scale of the possible systematic effects is not well known. The effect is probably less than 1 mas. Considering the random and possible systematic errors, the accuracy and stability of the radio reference frame is probably at the level of 1 to 2 mas. This level is sufficient for almost all current celestial and terrestrial studies requiring a fixed reference frame.

Catalogs generated using different reference days or different definitions of right ascension zero point will differ but only by small rotations related to the errors in the standard precession and nutation models and the choice of zero point definition. These rotations do not affect the relative 
angles between sources.

The connection to the optical frame is another, very complicated problem. The FK5 positions of some 40 optical counterparts of extragalactic radio sources have been determined from recent plates. These right ascensions can be used to set the zero point of the radio coordinate system. After the zero point is set, a group of well-behaved, compact radio sources (the IERS suggests about 20) can be used to maintain the stability of the zero point for further analyses and as more data are added. It should be emphasized, however, that the radio zero point is independent of the optical equinox origin and is set to the FK5 origin only to facilitate radio/optical comparisons. Likewise the pole of the radio catalog is completely independent of the pole of FK5.

\section{Future Prospects}

The current state of the radio reference frame is quite good, and the future looks even brighter. The geodetic programs organized by NASA, NGS, and USNO will continue, the latter two perhaps indefinitely because of their functions, so that a core set of sources will be continually measured. The goal of the NRL reference frame and southern observing programs is to locate a well distributed set of 400 radio sources with precision approaching 1 mas for maintaining the radio reference frame and for linking the optical FK5 frame to the radio frame. These observations will continue for several years using such networks that are appropriate and available. Coming into play in the next few years will be the Very Long Baseline Array, a set of 10 identical stations as far south and west as Hawaii, as far north as the state of Washington, and as far east as the Virgin Islands. While this facility is designed primarily for study of radio source structure, it will be available for astrometry and will also require astrometric calibration. It has the potential for becoming the primary, ongoing supplier of high quality astrometric data. The astrometric data base will continue to expand and must be preserved just as carefully as plates. It will then be possible to combine even more observing programs and to improve the analysis over time. Our group and the group at JPL are undertaking a cooperative effort in this direction. We have already included JPL Mark II data in some of our analyses, although not here, and JPL has begun to analyze IRIS and CDP data. We have compared our analysis software and will continue these comparisons as the models are improved. These efforts will give more refined limits on possible systematic and analysis errors.

\section{References}

Fanselow, J.L. et al. (1984) Astron. J. 89, 987.

Herring, T.A. et al. (1986) J. Geophys. Res. 91, 4745.

Ma, C. et al. (1986) Astron. J. 92, 1020.

Ma, C. et al. (1990) Astron. J., in press.

McCarthy, D.D. ed. (1989) IERS Standards (1989), Paris Observatory, Paris.

Robertson, D.S. (1975) Ph.D. thesis, Massachusetts Inst. of Technology.

Robertson, D.S. et al. (1986) Astron. J. 91, 1456.

Shapiro, I.I. (1967) Science 157, 806.

Sovers, O.J. et al. (1988) Astron. J. 95, 1647.

Sovers, O.J. (1990), in IAU Symposium 141, this volume. 


\section{Discussion}

YATSKIv: How many estimates of the zenith atmospheric delay per day are you including in your solution?

MA: At the very first stage only a single zenith atmospheric delay is employed. The final analysis is done using between 8 and 24 residual atmosphere parameters per station per day.

KAPLAN: Does your astrometric program include any galactic objects?

MA: Our astrometric program as currently conceived does not include any galactic objects. We would consider using suggested galactic objects, but such sources would need to be observable (at both $8.4 \mathrm{GHz}$ and $2.3 \mathrm{GHz}$ ) on our astrometric networks, which are generally considerably less sensitive than the astronomical VLBI networks.

WALTER: GSFC has published several catalogues of VLBI positions since the middle of the seventies. If new observations become available will then all observations be lumped together for setting up a successor catalogue, or do you confine yourself to the more recent data? Work on astronomical constants would be facilitated by the latter case which would for instance allow a better distinction of epochs of observation.

MA: The normal procedure of the Goddard VLBI group is to use all the data (both old and new) of acceptable quality available at the time to make each new source position catalog. In practice this means essentially all of our Mark III data (from fixed stations). There are also Mark I data from 1972-78, but these are not used because they lack dual frequencies for ionospheric calibration. It is simple to make catalogs using only data from specific time intervals, and these catalogs could be generated for studies of astronomical constants. The Mark I data, if somehow properly calibrated, might provide a useful extension of the time base for directly estimating the long-period astronomical constants, but they would not give much additional information for the source position catalog. It should be noted that source catalogs generated only from our geodetic data are much smaller than catalogs generated from our geodetic and astronomical data together. 